\title{
Bandwidth Improvement for Vibration Energy Harvesting devices
}

\author{
Jens Twiefel ${ }^{1,2}$, Marcus Neubauer $^{1,2}$, Jörg Wallaschek $k^{1,2}$ \\ ${ }^{1}$ Leibniz Universität Hannover, Inst. f. Dynamik und Schwingungen, Appelstr. 11, Hannover, Germany \\ twiefel@ids.uni-hannover.de \\ ${ }^{2}$ Leibniz Universität Hannover, Center for Energy Harvesting, Appelstr. 11, Hannover, Germany
}

\begin{abstract}
:
This contribution provides a technology overview on techniques to enlarge the bandwidth of vibration energy harvesting devices. In general a broadband device is demanded, such a device would be easily applicable on many vibration sources without any adoption. Up to date such a system is not available. However, improving the broadband characteristic has been investigated intensely in the recent years. This paper introduces vibration energy harvesting briefly and provides a detailed classification of the various broadband techniques.
\end{abstract}

Key words: Power Harvesting, Vibration, Broad Band Energy Harvesting, Piezoelectric

\section{Introduction}

Energy harvesting or energy scavenging is utilizing (parasitic) energy form the (direct) environment to power useful applications. The definition may be extended by the requirement, that the harvesting system has no significant feedback to the host system.

The concept of energy harvesting allows creating autonomous devices which can run maintenance free for the complete product lifetime. This advantage leads directly to a second important aspect; the system is capable of full capsulation which is a requirement for harsh environments. A broad overview on energy harvesting technologies is given in [1]

A typical energy harvesting system consists of five main components (Fig. 1): energy conversion, energy conditioning, energy storage, power management, and the application.

For the energy conversion all available energy forms may be used, in industrial environments most often mechanical, thermal, and radiation energy is available. In typical industrial indoor applications, the first two have the highest potential. In this environment piezoelectric transducer are providing the highest power density [2].

The energy conditioning is needed to match the impedances between the transducer and the storage and to rectify AC Voltages into DC.

To ensure that there is no feedback to the host system the harvested amount of energy is ra- ther small, in the area of a few $\mathrm{mW}$. To perform complex tasks an energy storage and power management is essential.

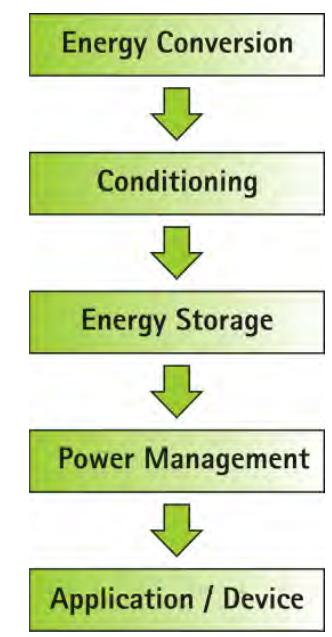

Fig. 1. Components of an energy harvesting system

The application is certainly the most important component of the harvesting system. Wireless sensor nodes are most promising for industry automation. Such nodes can be places in suited locations on machinery, especially on moving parts or other hard to reach areas. Further there is a possible impact on the cost due to dramatically reduced mounting effort, compared to traditional wired sensor nodes.

The design of energy harvesting systems is a complex task. To ensure a good performance, all components needs to exhibit high efficiency. 


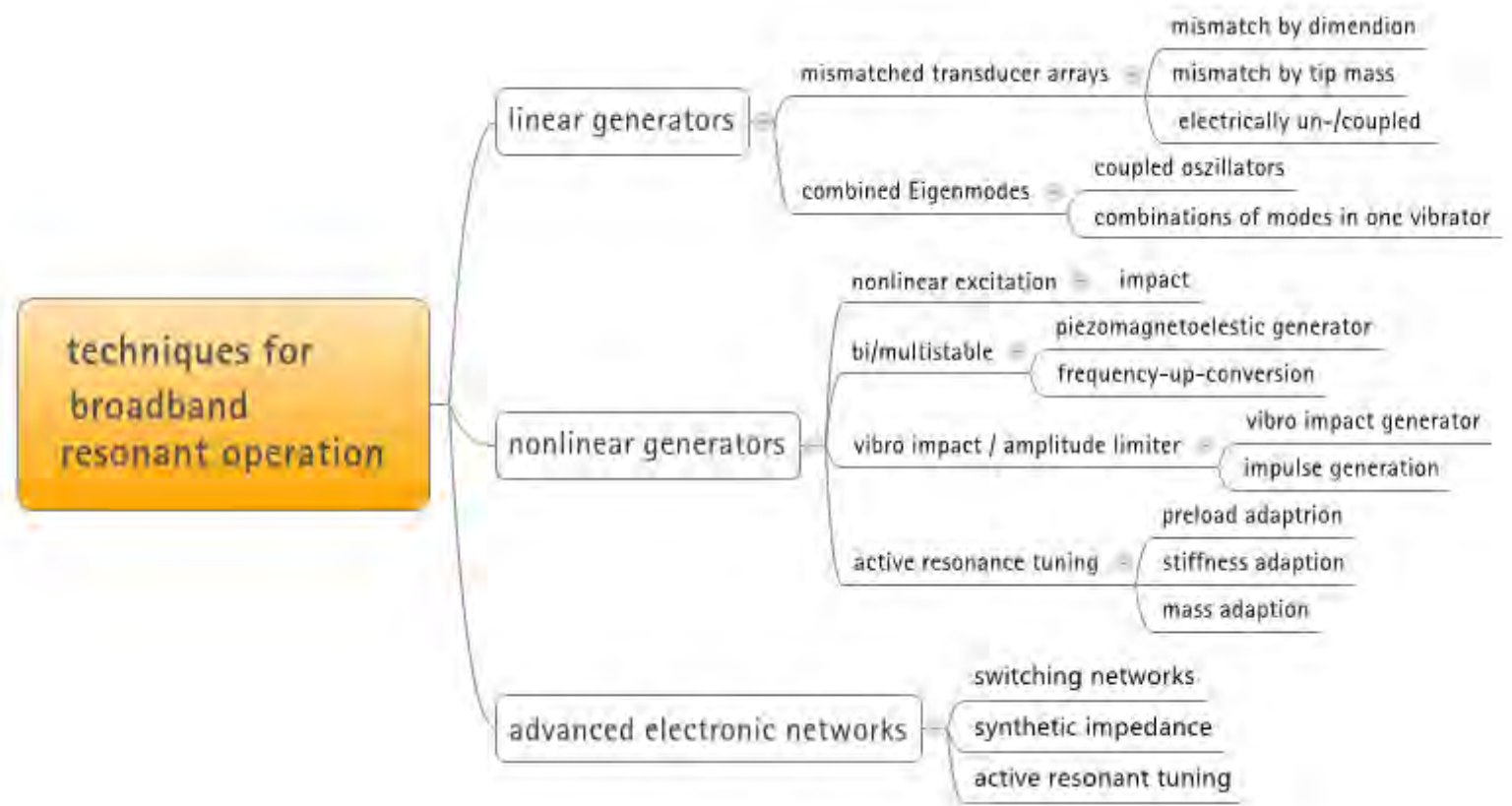

Fig. 2. Classification of broadband techniques.[3]

For the here focused component, the energy conversion from mechanical vibration at the example of piezoelectric transducers. Two basic operation modes can be distinguished: quasi static and resonant operation.

A transducer for quasi static operation is used far below its first relevant eigenfrequency. In the FRF, the output (per cycle) is nearly constant in the quasi static frequency range. Consequently the bandwidth is huge, but the strain dependent energy output is low. This leads to bulky devices.

The alternative is utilizing the resonance peak. Here the transducer is designed to have its first eigenfrequncy at the major frequency from the target environment. In resonance the transducer performs high vibration amplitudes for small input excitation. To maximize the output, the inner damping of the transducer needs to be minimized. However, if a transducer provides a sharp, high resonance peak its frequency dependence is high (small bandwidth). This makes an application dependent design and a low tolerance fabrication necessary. The size of transducers well on the target environment adopted is much smaller than the size of a comparable quasi static transducer.

In a consequence resonant transducers are wanted for energy harvesting applications. But a major improvement in bandwidth is needed to allow all-purpose devices. Such are the key to bring energy harvesting technology into the makes at reasonable lot sizes and at completive prices.
Much work has been done in recent years with this purpose by many researchers. In the next section those ideas are sorted and classified. The principals are explained and their advantages and drawbacks are pointed out.

\section{Techniques for bandwidth improvement}

To improve the bandwidth of vibration harvester without decreasing the power output different technologies have been investigated. To cluster those, tree major groups are proposed [3]: linear generators, non-linear generators and advanced electronic networks.

In the group linear generators either multiple transducers or used combined eigenmodes are used to improve the bandwidth. Those techniques may be modeled by means of linear theory.

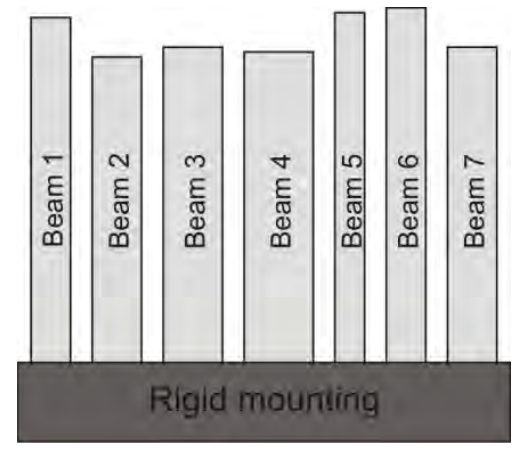

Fig. 3. Array of piezoelectric transducers

Belong them mismatched generator arrays can provide an improved bandwidth. Multiple transducers with slightly different resonance frequencies are mounted in one device, i.e. [5-7]. 
The basic idea is to have always one among them operating in its resonance even if the major frequency of the target environment changes. To ensure a high output power, the individual transducers cannot be coupled electrically. An electrical coupling would allow energy flow between them [4]. Typically, this decoupling is carried out by individual rectifiers for each transducer of the array. Mechanical coupling between the transducer is mostly neglected by the assumption of a rigid mounting.

A second possibility is the combination of eigenmodes of one structure, i.e. [8-12]. The structure is designed in a way that two or more of its eigenfrequencies are nearby, to increase the working range. Similar to the mismatched arrays the electrical coupling needs to be considered. Further the mechanical interaction is not longer negligible.

In general the design of linear generator techniques is challenging in terms of reproducibility due to the high parameter dependence of the performance. A drawback is the high number of parts, piezoelectric transducer as well as circuitry components.

Further it is questionable if the increased bandwidth could be also reached by combining all used piezoelectric material for one single transducer [4], with even better performance.

Non linear generators group the techniques which require non-linear models for their description.

One possibility is the use of impacts or shocks to excite the transducer, i.e. [13-16]. An impact excites all resonances of the transducer. The vibration will be used to harvest the energy. If the impact excitation at a low frequency the technique is sometimes called frequency-up conversion. A rather simple setup may be found in a patent [17] from 1971. This technique was also employed in the piezoelectric wireless light switches by enOcean [18]. It is especially advantageous if the energy is needed rather seldom like those switching applications.

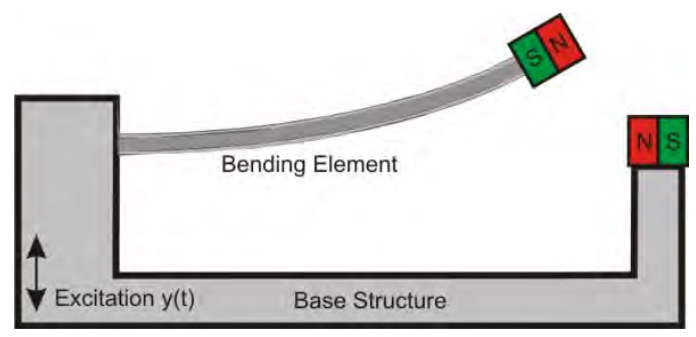

Fig. 4 Setup of a bistable system

A similar mechanism is used in bistable or multistable energy harvesters [19-26]. Resonate vibration is excited by switching from one stable position into another. Especially low frequency vibrations with high amplitudes are well suited to initiate the switching process. This technique creates wide bandwidth for a wide frequency range at low frequencies, which is a desire in many possible application scenarios. A typical setup is given in Fig. 4 .

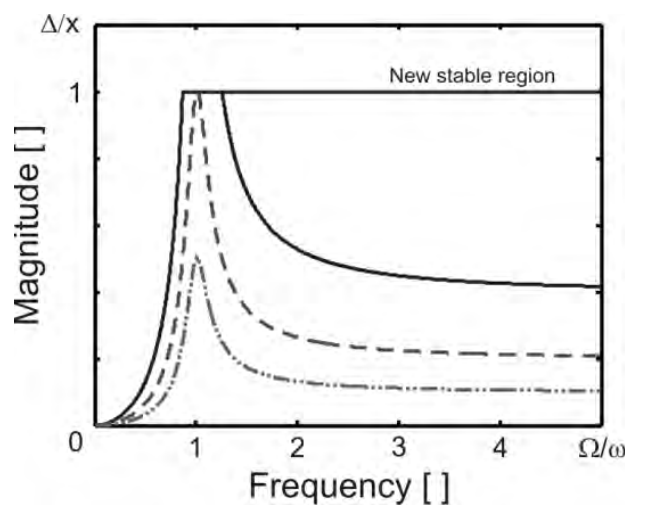

Fig. 5 Ideal frequency response of a vibro-impact harvester with rigid stoppers

Vibro-impact generators are capable to extend the bandwidth towards higher frequencies, i.e. [27-32]. Here the free vibration amplitude is limited by mechanical stoppers. In the majority of the investigations, the stoppers are assumed to be rather rigid and the vibrating element is the transducer. In some cases the stoppers are the transducer. However, as soon the vibration amplitude is high enough to reach the stopper in resonance the frequency response becomes nonlinear. Fig. 5 shows this basic idea. From this amplitudes a new stable plateau emerge, and the bandwidth is increased dramatically for higher excitation frequencies. However, reaching the plateau is the challenge, which is only possible near resonance or with huge amplitudes. Further the stable plateau is sensitive to disturbances (reduced input amplitude), so that the system easily can "drop down". Hence, the vibro-impact technique essential need a defined start up of the host system, maybe something like the run-up of a turbine or engine.

The ideal Fig. 5 implied that the bandwidth has no upper restriction, which is only correct for an ideal 1-dof system. However, even for a real system more than $10 \%$ of the resonance frequency seems to be a possible bandwidth. This is still a huge improvement.

The adaptive tuning of the transducer is the last, in Fig. 2 mentioned technique. Here the physical properties of the transducer are changed by various means [33-39]. Moving the tip mass of a cantilever beam would be the easiest thought. The challenge in the resonance is the energy need and the mechanism to change the property. Mechanical adoption is only possible by means of actuators; therefore 
a high (for an energy harvesting system) demand of energy exists. The major application for the adaptive tuning is not the real-time tracking of the environment frequency, but a slow (after major changes) adaption is reasonable.

The third group the possibilities in the electrical domain, the advanced electronic networks.

Switching networks have gained a lot of attention. Especially the SSHI (synchronized switched harvesting on inductance) technique is focused in [40-45]. On the electrical side of the transducer a new branch is connected. This consist of an inductor, a resistor, and most important a switch. Using the switch, the charge on the piezoelectric element can be inverted. A suited switching law guaranties that this is done at the right moment. The studies show that the working frequency range can be enlarged significantly. However, getting the data for the switching decision and the needed power for the data processing and switching is a major issue. But it has been shown, that a harvester can power its own circuitry.

\section{Conclusion}

All presented and classified techniques have their application fields none of them is a general solution for the all-purpose energy harvesting device. Hence many target environments cannot provide stable vibration parameters (major frequency, or amplitude) a broadband system is necessary. If the frequencies are low a frequency-up-conversion using bistable generators is a good choice. For user operated elements such as switches the impact excitation is a good selection. In case the environment parameters are pretty stable and predictable an irregularly adoption is well suited. The vibroimpact systems have their applications where the bandwidth needs to be extended to higher frequencies.

\section{References}

[1] Priya, Shashank, and Daniel J. Inman, eds. Energy harvesting technologies. Springer, 2008.

[2] Roundy, Shad, et al. "Improving power output for vibration-based energy scavengers." Pervasive Computing, IEEE 4.1 (2005): 28-36.

[3] Twiefel, Jens, and Henrik Westermann. "Survey on broadband techniques for vibration energy harvesting." Journal of Intelligent Material Systems and Structures (2013).

[4] Neubauer, Marcus, et al. "Modeling Aspects of Nonlinear Energy Harvesting for Increased Bandwidth." (2012).

[5] Liu, Jing-Quan, et al. "A MEMS-based piezoelectric power generator array for vibration energy harvesting." Microelectronics Journal 39.5 (2008): 802-806.

[6] Ferrari, Marco, et al. "Piezoelectric multifrequency energy converter for power harvesting in autonomous microsystems." Sensors and Actuators A: Physical 142.1 (2008): 329-335.

[7] Xue, Huan, Yuantai Hu, and Qing-ming Wang. "Broadband piezoelectric energy harvesting devices using multiple bimorphs with different operating frequencies." Ultrasonics, Ferroelectrics and Frequency Control, IEEE Transactions on 55.9 (2008): 2104-2108.

[8] Erturk, Alper, Jamil M. Renno, and Daniel J. Inman. "Modeling of Piezoelectric Energy Harvesting from an L-shaped Beam-mass Structure with an Application to UAVs." Journal of intelligent material systems and structures 20.5 (2009): 529544.

[9] Castagnetti, D. "Experimental modal analysis of fractal-inspired multi-frequency structures for piezoelectric energy converters." Smart Materials and Structures 21.9 (2012): 094009.

[10] Bartsch, U., J. Gaspar, and O. Paul. "Lowfrequency two-dimensional resonators for vibrational micro energy harvesting." Journal of Micromechanics and Microengineering 20.3 (2010): 035016.

[11] Abdelkefi, A., et al. "Energy harvesting from a multifrequency response of a tuned bendingtorsion system." Smart Materials and Structures 21.7 (2012): 075029.

[12] Qi, Shaofan, et al. "Design of a multiresonant beam for broadband piezoelectric energy harvesting." Smart Materials and Structures 19.9 (2010): 094009

[13] Renaud, Michael, Paolo Fiorini, and Chris van Hoof. "Optimization of a piezoelectric unimorph for shock and impact energy harvesting." Smart materials and structures 16.4 (2007): 1125.

[14] Renaud, Michael, et al. "Harvesting energy from the motion of human limbs: the design and analysis of an impact-based piezoelectric generator." Smart Materials and Structures 18.3 (2009): 035001.

[15] Cavallier, B., et al. "Energy harvesting using vibrating structures excited by shock." Ultrasonics Symposium, 2005 IEEE. Vol. 2. IEEE, 2005.

[16] Gu, Lei, and Carol Livermore. "Impact-driven, frequency up-converting coupled vibration energy harvesting device for low frequency operation." Smart Materials and Structures 20.4 (2011): 045004.

[17] Honig, William. "TRANSMITTER PIEZOELECTRIC POWER SUPPLY." U.S. Patent No. 3,553,588. 5 Jan. 1971.

[18] Grassl, Erwin, and Frank Schmidt. "Energyautonomous electromechanical wireless switch." U.S. Patent No. 7,019,241. 28 Mar. 2006. 
[19] Erturk, A., and D. J. Inman. "Broadband piezoelectric power generation on high-energy orbits of the bistable Duffing oscillator with electromechanical coupling." Journal of Sound and Vibration 330.10 (2011): 2339-2353.

[20] Cohen, Nadav, Izhak Bucher, and Michael Feldman. "Slow-fast response decomposition of a bistable energy harvester." Mechanical Systems and Signal Processing (2012).

[21] Westermann, Henrik, Marcus Neubauer, and Jörg Wallaschek. "Bandbreitensteigerung von piezoelektrischen Energy Harvesting Systemen durch Magnetkräfte." at-Automatisierungstechnik 60.7 (2012): 384-391.

[22] Harne, R. L., and K. W. Wang. "A review of the recent research on vibration energy harvesting via bistable systems." Smart Materials and Structures 22.2 (2013): 023001.

[23] Arrieta, A. F., et al. "A piezoelectric bistable plate for nonlinear broadband energy harvesting." Applied Physics Letters 97.10 (2010): 104102104102.

[24] Stanton, Samuel C., Clark C. McGehee, and Brian P. Mann. "Nonlinear dynamics for broadband energy harvesting: investigation of a bistable piezoelectric inertial generator." Physica D: Nonlinear Phenomena 239.10 (2010): 640-653.

[25] Liu, W. Q., et al. "Novel piezoelectric bistable oscillator architecture for wideband vibration energy harvesting." Smart Materials and Structures 22.3 (2013): 035013.

[26] Andò, B., et al. "Nonlinear mechanism in MEMS devices for energy harvesting applications." Journal of Micromechanics and Microengineering 20.12 (2010): 125020.

[27] Moss, Scott, et al. "A low profile vibro-impacting energy harvester with symmetrical stops." Applied Physics Letters 97 (2010): 234101.

[28] Soliman, M. S. M., et al. "A wideband vibrationbased energy harvester." Journal of Micromechanics and Microengineering 18.11 (2008): 115021.

[29] Mak, K. H., et al. "Vibro-Impact Dynamics of a Piezoelectric Energy Harvester." Structural Dynamics and Renewable Energy, Volume 1 (2011): 273-280.

[30] Liu, Huicong, et al. "Investigation of a MEMS piezoelectric energy harvester system with a frequency-widened-bandwidth mechanism introduced by mechanical stoppers." Smart Materials and Structures 21.3 (2012): 035005.

[31] Ferrari, M., et al. "Impact-Enhanced Multi-Beam Piezoelectric Converter for Energy Harvesting in Autonomous Sensors." Procedia Engineering 47 (2012): 418-421.

[32] Tvedt, Lars Geir Whist, Duy Son Nguyen, and Einar Halvorsen. "Nonlinear behavior of an electrostatic energy harvester under wide-and narrowband excitation." Microelectromechanical Systems, Journal of 19.2 (2010): 305-316.
[33] Wu, W., et al. "Tunable resonant frequency power harvesting devices." Proceedings of SPIE, the International Society for Optical Engineering. Vol. 6169. Society of Photo-Optical Instrumentation Engineers, 2006.

[34] Challa, Vinod R., et al. "A vibration energy harvesting device with bidirectional resonance frequency tunability." Smart Materials and Structures 17.1 (2008): 015035.

[35] Ayala, Ivo N., et al. "Autonomous tunable energy harvester." Proc. PowerMEMS (2009): 49-52.

[36] Wischke, M., et al. "Electromagnetic vibration harvester with piezoelectrically tunable resonance frequency." Journal of Micromechanics and Microengineering 20.3 (2010): 035025.

[37] Peters, Christian, et al. "A closed-loop wide-range tunable mechanical resonator for energy harvesting systems." Journal of Micromechanics and Microengineering 19.9 (2009): 094004.

[38] Ferrari, M., et al. "Improved energy harvesting from wideband vibrations by nonlinear piezoelectric converters." Sensors and Actuators A: Physical 162.2 (2010): 425-431.

[39] Roundy, Shad, and Yang Zhang. "Toward selftuning adaptive vibration-based microgenerators." Proc. SPIE. Vol. 5649. 2004.

[40] Guyomar, Daniel, et al. "Toward energy harvesting using active materials and conversion improvement by nonlinear processing." Ultrasonics, Ferroelectrics and Frequency Control, IEEE Transactions on 52.4 (2005): 584-595.

[41] Lien, I. C., et al. "Revisit of series-SSHI with comparisons to other interfacing circuits in piezoelectric energy harvesting." Smart Materials and Structures 19.12 (2010): 125009.

[42] Lallart, Mickaël, et al. "Double synchronized switch harvesting (DSSH): a new energy harvesting scheme for efficient energy extraction." Ultrasonics, Ferroelectrics and Frequency Control, IEEE Transactions on 55.10 (2008): 2119-2130.

[43] Neubauer, M., M. Krack, and J. Wallaschek. "Parametric studies on the harvested energy of piezoelectric switching techniques." Smart Materials and Structures 19.2 (2009): 025001.

[44] Neubauer, Marcus, Xu Han, and Sebastian M. Schwarzendahl. "Enhanced switching law for synchronized switch damping on inductor with bimodal excitation." Journal of Sound and Vibration 330.12 (2011): 2707-2720.

[45] Ottman, Geffrey K., et al. "Adaptive piezoelectric energy harvesting circuit for wireless remote power supply." Power Electronics, IEEE Transactions on 17.5 (2002): 669-676. 